This manuscript is a preprint and has not been submitted for publication in a journal. Please feel free to contact the first author; we welcome feedback. 


\section{Detection and temperature estimation of gas flares with nocturnal Landsat OLI}

Ruiwen Lee ${ }^{\mathrm{a}}$ and Christopher Small ${ }^{\mathrm{b}}$

${ }^{a}$ School of International and Public Affairs, Columbia University, New York, NY, USA

${ }^{b}$ Lamont-Doherty Earth Observatory, Columbia University, Palisades, NY, USA

ruiwen.lee@columbia.edu. http://orcid.org/0000-0002-3455-9916. School of International and Public Affairs, Columbia University, 420 W 118th St \#1410, New York, NY 10027, USA. 


\section{Detection and temperature estimation of gas flares with nocturnal Landsat OLI}

Natural gas flaring is a worldwide polluting activity carried out during oil production. Satellite imagery has emerged as a low-cost, objective tool to measure and monitor gas flaring. Since 2012, hectometre-resolution infrared imagery from the Suomi NPP VIIRS sensor has been used to operationally monitor global gas flaring (Elvidge et al. 2016). Since 2013, nocturnal acquisitions of Landsat 8 OLI and TIRS imagery have been collected intermittently in a small number of locations worldwide - including part of the Williston basin in North Dakota, where oil production from shale formations results in an unusually high density of production wells. The goal of this study is to compare the use of VIIRS and nocturnal Landsat 8 Operational Land Imager (OLI) in identifying and characterizing gas flare populations associated with tight oil production, where flares are smaller, more numerous, and more closely spaced than in conventional oil production. We find that the OLI optical bands resolve individual closely-spaced $(<200 \mathrm{~m})$ flares, and that most flares in this region are small and persistent on time scales of months. Most of these flares are not detected by either the VIIRS Day/Night Band or NOAA's VIIRS Nightfire product. Of the 154 largest flares selected, 73 contained saturated pixels in at least one OLI band. We invert the Planck function for blackbody radiation to estimate temperature and emissivity for the 81 non-saturated flares. Temperature estimates are plausible (1500 K to $2200 \mathrm{~K})$, but RMS misfits vary by orders of magnitude. The Nightfire product detects and provides temperature estimates for 16 of these 81 flares. OLI-derived temperatures are comparable to Nightfire estimates, but generally higher. Although Landsat's 16-day revisit period precludes its use for flare monitoring on its own, the positional accuracy and low noise floor of OLI SWIR bands allows for repeat imaging of individual flares in settings where most flares are too small and closely-spaced to be resolved by VIIRS. Because these small flares account for most of the emission from tight oil production, nocturnal Landsat could provide an important complement to flaring estimates derived from VIIRS alone.

\section{Introduction}

Since the tight oil boom began in the mid-2000s, the night skies of North Dakota are no longer dark. Bright flares light up what used to be a predominantly agricultural landscape as everyday, 
millions of cubic metres of raw natural gas, which is extracted together with oil, is burnt away almost immediately after it surfaces (NDIC 2019). People from other oil-producing nations across the globe observe a similar sight. Gas flaring is a large-scale polluting activity carried out worldwide during oil production. Based on VIIRS data, the World Bank estimates that in 2017, more than 140 billion cubic metres $(\mathrm{bcm})$ of natural gas was flared worldwide. Not only was its energy not put to use, more than 300 million tons of $\mathrm{CO}_{2}$ were emitted into the atmosphere. Knowing where, when and how much natural gas is flared will become increasingly important if monitoring emission sources becomes common practice.

Satellite imagery has emerged as a low-cost, objective tool to measure and monitor gas flaring. Elvidge et al. (2009) first estimated national and global gas flare volumes with satellite observations of night light. They used 15 years of night light imagery from the Defense Meteorological Satellite Program (DMSP) Operational Linescan System (OLS), and estimated that global flared volume was between 140 to $170 \mathrm{bcm}$ annually over the 1994-2008 study period. Subsequent studies used ATSR thermal (Casadio, Arino, and Serpe 2012), MODIS thermal (Anejionu, Blackburn, and Whyatt 2015), and VIIRS SWIR (Elvidge et al. 2016). All these products are adequate for monitoring large flares at the national or regional level because their wide swaths provide synoptic coverage of large areas with near daily revisit periods. However, the swath-resolution trade-off limits the spatial resolution of these sensors to $>750 \mathrm{~m}$ at nadir with significantly diminishing resolution approaching swath edges. Day time Landsat imagery has been shown capable of detecting large gas flares (Anejionu, Blackburn, and Whyatt 2014; Chowdhury et al. 2014; Fisher and Wooster 2018), but the 16-day revisit period precludes its use for operational monitoring. Elvidge et al. (2015) show that nocturnal Landsat SWIR and TIR bands can be used to differentiate between flaming and smouldering combustion phases of 
peat fires in Indonesia. However, to our knowledge, nocturnal Landsat imagery has not been used for identification of small gas flares in settings where wells are too closely-spaced to be resolved by hectometre-resolution imagery.

Compared to the hectometre-resolution sensors used for operational monitoring, Landsat provides higher spatial resolution with the potential to resolve individual flares in settings where larger numbers of smaller flares are common. Tight oil production typically requires larger numbers of much more closely spaced wells with smaller flares than those associated with conventional oil production. As such, hectometre-resolution sensors, like VIIRS, may not detect smaller flares and therefore may miss a significant source of flared gas. The objective of this study is to use night time Landsat imagery to characterize gas flares in an area with denselyspaced wells and small flares, and to draw comparisons with the capabilities of VIIRS, the predominant satellite used to monitor gas flaring. As observed by Elvidge et al. (2019), VIIRS DNB detects many more night time fires than VIIRS Nightfire, which is a product that includes fire temperature estimates. We thus compare Landsat's flare detections against VIIRS DNB's, and compare Landsat temperature estimates against Nightfire's in this paper. While Landsat's 16-day revisit is not adequate for operational monitoring, its higher spatial resolution may provide independent verification of VIIRS flare estimates at specific times and locations, and it may also provide estimates of small flare populations not resolved by VIIRS in dense well settings associated with tight oil production.

\section{Methods}

\subsection{Data and study area}

Landsat 8 (L8) acquisitions are downloaded from the United States Geological Survey's (USGS) EarthExplorer website (http://earthexplorer.usgs.gov). The L8 data used in our primary analysis 
are from the optical bands (1-7), collected by the Operational Land Imager (OLI). Daily VIIRS data is downloaded from the National Aeronautics and Space Administration's (NASA) Level-1 and Atmosphere Archive \& Distribution System (LAADS) Distributed Active Archive Center (DAAC). We also obtained VIIRS Day/Night Band (DNB) monthly composites (Elvidge et al. 2017) and VIIRS Nightfire data (Elvidge et al. 2016) produced by the National Oceanic and Atmospheric Administration's (NOAA) Earth Observation Group (EOG) (https://ngdc.noaa.gov/eog/viirs).

We use nine relatively cloud-free nocturnal L8 acquisitions in 2016-2017 of western North Dakota, where thousands of oil and gas wells are in operation (NDIC 2018). Figure 1 shows day time L8 and night time VIIRS DNB acquisitions from the study area within the study time frame. The most conspicuous geographical feature in this area is the Missouri River, which roughly divides the study area into two halves, north and south. Of the nine cloud-free nocturnal L8 acquisitions, five are from south of the river (path 126 row 217 on 9 May 2016, 26 June 2016, 14 September 2016, 16 October 2016, and 3 December 2016), and four are from north of the river (path 126 row 218, on 9 May 2016, 14 Sep 2016, 16 October 2016, and 10 April 2017). The inset VIIRS image shows a tri-temporal composite of monthly mean luminance, illustrating the spatiotemporal variability of flaring in the basin.

[Figure 1 approximately here]

Comparison of near-coincident acquisitions of Landsat 8 and VIIRS illustrates the benefit of decametre resolution in areas with high densities of smaller flares. Figure 2 compares an OLI infrared band composite (top) with a coregistered OLI SWIR and VIIRS DNB composite (centre) and a VIIRS TIR/MIR/SWIR composite (bottom) for the most densely drilled part of the study area south of the Missouri River on 9 May 2016. OLI imaged the study area at 04:25 GMT 
and VIIRS imaged it at 10:24 GMT. Also shown are locations of NOAA Nightfire product detections for the same night. The OLI IR composite is displayed as $\log _{10}$ radiance with a linear contrast stretch between the band-specific non-zero minimum and maximum radiance. The largest flares stand out against the noise background with the SWIR2 band imaging a larger halo than the NIR band as a combined result of the lower SWIR2 noise floor (explained below) and the higher SWIR emission of the flares. Smaller flares recede into the noise floor in the OLI IR composite but are clearly visible in the OLI SWIR + VIIRS DNB composite (centre) when the OLI bands are stretched from the noise floor to maximum radiance. When viewed at full resolution, it is immediately apparent that OLI resolves many more small flares than VIIRS DNB, and that many of the $750 \mathrm{~m}$ VIIRS DNB pixels image multiple flares. In contrast, the VIIRS TIR/MIR/SWIR composite resolves many fewer flares in the SWIR and only the few largest flares (or the heated ground surface surrounding the flare) in the TIR. The NOAA Nightfire product detects all of the flares visible in the SWIR band of the composite, as well as several others that are not obvious in the full range linear stretch. It is clear that OLI's $30 \mathrm{~m}$ resolution provides a far more detailed depiction of the flare population in areas with denselyspaced wells and small flares.

[Figure 2 approximately here]

\subsection{Characterization of flares using Landsat 8 imagery}

Emitting at high temperatures from around $1300 \mathrm{~K}$ to $2700 \mathrm{~K}$, gas flares are clearly observable at SWIR wavelengths where their emitted radiance peaks. Flares can sometimes be observed in the TIR against the cool ground surface at night. Figure 3(a) compares the full resolution L8 optical

(left) and thermal (right) imagery from a section around the Missouri River. The optical image is a composite of the NIR, SWIR1 and SWIR2 bands (OLI bands 5-7) while the thermal image 
displays the TIR band (thermal band 10) in grayscale. Emitting sources can be seen clearly in the optical IR composite. To verify that these are flares associated with oil production, we examine twelve (groups of) hotspots, labelled A through L on the optical IR composite. Metre-resolution day time visible images of these hotspot locations in Figure 3(b) show clearly that oil/gas wells or gas processing facilities exist at these sites, and that gas flares can indeed be seen in most of them. The decametre spatial resolution of Landsat images allows flares near each other to be distinguished (for example, locations $\mathrm{G}$ and $\mathrm{K}$ in Figure 3(b)).

[Figure 3 approximately here]

The radiance distributions for the full flare population illustrate the large number of flares and the wide range of sizes and radiances. Figure 4 shows band-specific bivariate distributions of flare radiance and area (diagonal) for the dense flare region shown in Figure 2. We refer to the size (or area) of a flare in the context of the spatially contiguous area of pixels with radiance levels above the noise floor for that spectral band. While the flares themselves are obviously smaller than $30 \mathrm{~m}$ diameter, the combination of scattering, ground reflection and adjacency effect results in signatures spanning several pixels in most cases. Flare area is calculated as the total area of each spatially contiguous (Rook's case) cluster of pixels segmented using the bandspecific noise floor as a threshold. To avoid spurious detections, only clusters 4 pixels and larger are considered. Band-specific noise floors and peak radiance wavelengths are clearly evident in the band pair scatterplots above the diagonal. Example Planck distribution fits to individual pixel spectra for several of the largest flares are shown below the diagonal. The spectrum of each flare is shown with Planck distribution fits (described below) on three dates in 2016. Although the fits are shown on semilog plots for clarity, the temperature and emissivity estimates were made from untransformed at-sensor radiances. Together, the plots show the varying noise floors and peak 
radiances for the OLI IR bands, the asymptotic increase in peak radiance with flare area, and the generally plausible fits obtained for flares with peak radiances spanning $\sim 3$ orders of magnitude.

[Figure 4 approximately here]

\subsection{Estimating flare temperatures using Planck inversion}

The flares with OLI radiance significantly larger than the band-specific noise floor generally peak in SWIR bands 6 or 7, suggesting that it may be possible to estimate their temperatures by inversion of the Planck distribution function for blackbody radiation. While Fisher and Wooster (2018) have shown that the MIR-radiance method can be modified for higher temperature sources with peak emissions at SWIR wavelengths to return unbiased estimates of Fire Radiative Power (FRP), we apply the Planck inversion approach used by Elvidge et al. (2013) for consistency with the Nightfire flare temperature estimates. Since flare radiance peaks in the optical IR bands, we first select 154 bright hotspots (clusters of pixels) clearly visible in the SWIR1 (OLI band 6). We then select the pixel with the highest SWIR1 radiance within each hotspot for analysis. In this paper, we refer to these 154 pixels as flares for simplicity. Using the online atmospheric correction parameter calculator described in Barsi, Barker and Schott (2003), we find that atmospheric transmissivity during acquisition times was fairly high, ranging from 0.80 to 0.96 . We thus use Top of Atmosphere (ToA) radiances for the optical bands. Also, in the context of VIIRS data, Elvidge et al. (2016) confirm that atmospheric effects are small at the SWIR wavelengths.

When saturation occurs in pixels imaging a flare or near a flare, the cubic convolution resampling method used to process L8 images can result in the saturated pixels appearing dark within the cluster of bright pixels (J. Barsi, personal communication, July 18, 2017), rather than taking on the maximum DN value. Schroeder et al. (2016) and Fisher and Wooster (2018) also 
describe pixel saturation in OLI SWIR bands in the contexts of active fire detection and fire radiative power retrieval respectively. We verify that the pixels used for temperature estimates are not saturated, using the quality assessment band provided with the L8 Collection 1 Level-1 product. Of the 154 flares selected for study, 73 are saturated in at least one band, leaving 81 flares for temperature estimation.

We invert the Planck function for blackbody radiation to estimate temperature and emissivity using the optical bands for each flare. Planck's law is given by Equation (1):

$$
L_{\lambda}=\frac{2 h c^{2}}{\lambda^{5}} \frac{1}{e^{\frac{h c}{\lambda k_{\mathrm{B}} T}}-1}
$$

where $L_{\lambda}$ is the spectral radiance at wavelength $\lambda, T$ is the absolute temperature of the body, $h$ is the Planck constant, $c$ is the speed of light, and $k_{\mathrm{B}}$ is the Boltzmann constant.

The inversion was implemented using the fminsearch function in Matlab. The misfit is the Euclidean norm of the distance between the observed flare radiances and the Planck distribution radiances, summed over the optical bands; Equation (2) gives the misfit:

$$
m=\|\widehat{\boldsymbol{L}}-\boldsymbol{L}\|
$$

where $m$ is the misfit, $\mathbf{L}$ is the vector of observed radiances from the seven optical bands, and $\hat{\mathbf{L}}$ is the corresponding vector of radiances resulting from the inversion. We then use the mean squared misfit to calculate the standard RMSE:

$$
r=\sqrt[2]{\frac{1}{7} m^{2}}
$$

Since RMSE could increase mechanically with observed radiance, which differs by an order of magnitude across the pixels, we check if normalizing the RMSE affects our results. We also 
perform inversions using only the infrared optical bands as the higher noise floor and greater susceptibility to atmospheric scattering of the visible bands could compromise the inversion. Comparisons of both inversion results and description of the RMSE normalization are given in Appendix A.

\section{Results}

\subsection{Temperature estimates from Planck inversion}

The Planck inversion yields temperature estimates between 1500-2200 K, within the expected range for gas flares. However, a range of misfits are observed; Figure 5 presents examples of some of the best fits, and Figure 6 shows examples of some poor fits. RMSE of the fitted curves are generally an order of magnitude lower than peak radiance. Distributions of temperature, emissivity, and misfit are given in Appendix A.

[Figure 5 approximately here]

[Figure 6 approximately here]

\subsection{Comparison with VIIRS}

When nocturnal L8 OLI SWIR images are co-registered with VIIRS DNB radiance acquired on the same night (Figure 2, centre), we find that all night light detections above the DNB noise floor (except the city of Williston) coincide with high SWIR radiance in OLI, but most OLI detections are not resolved by VIIRS DNB. In many cases, multiple hotspots occur within a single VIIRS pixel, indicating that VIIRS' $750 \mathrm{~m}$ resolution is generally too coarse to identify individual wells and therefore not suitable for monitoring individual flares in tight oil producing areas, where well pads are often closely spaced. 
Flare temperatures estimated from OLI tend to be higher than NOAA Nightfire estimates. Figure 7 plots Nightfire temperature estimates against L8 temperature estimates for the 16 flares in our study with corresponding Nightfire detections on the same dates. We were only able to use a small number of flares for this comparison, because for many of the L8 flares, Nightfire either does not detect them or does not provide temperature estimates. Since the size of VIIRS pixels ranges from $750 \mathrm{~m}$ at nadir to $1600 \mathrm{~m}$ at the scan edge, we superimposed circles of $375 \mathrm{~m}$ radius and $800 \mathrm{~m}$ radius centred on the Nightfire detection locations on our selected L8 flares to identify the Nightfire detections that correspond to the L8 flares. Wells in the study area are often closely spaced, so many of our L8 flares have multiple, sometimes overlapping, Nightfire detection circles nearby. In such cases, we select the Nightfire detection with the closest location to the L8 flare for comparison. All pairs of L8 flares and Nightfire detections were retained only if we do not observe large neighbouring hotspots in the L8 SWIR1 band that lie within $800 \mathrm{~m}$ of the same overlaying Nightfire detection. In Figure 7, the red points represent isolated flares - L8 flares that only have one overlaying Nightfire detection and no neighbouring hotspots. The green points represent flares that have multiple Nightfire detections nearby, or small neighbouring hotspots within the overlaying Nightfire detection. Whether considering only the isolated flares (red points in Figure 7) or all flares with corresponding Nightfire detections (red and green points in Figure 7), we observe that the L8 estimates are generally higher than the Nightfire estimates. This is consistent with the much larger IFOV of VIIRS compared to Landsat 8 OLI. If the OLI's smaller IFOV yields more accurate temperature estimates than VIIRS, this may suggest that sensor spatial resolution and spatial density of wells could be important factors for further investigation in estimations of flare volume based on flare temperature. For instance, both Anejionu, Blackburn, and Whyatt (2015), and Elvidge et al. (2016) use statistical methods 
to calibrate gas flare temperatures to reported flare volumes. Future work developing a comprehensive dataset of estimated flare temperatures from Landsat could provide a complementary source of calibration for existing satellite products utilizing lower-resolution imagery.

[Figure 7 approximately here]

\subsection{Flare persistence and area}

The temporal persistence and size distribution of the flare population is illustrated by comparing three of the acquisitions from 2016 for path/row 126/217. Scatterplots of flare area for each date pair are shown in Figure 8. Each scatterplot shows the subset of flares detected in the same location on both dates. Distributions of flare areas, including those present on only one or two dates, are obtained using a decision tree classification (inset top) of the segmented areas of spatially contiguous pixels above the noise thresholds described above. It is evident that the vast majority of multiply-detected flares are at the small end of the size distribution, with a few flares either growing or shrinking between dates, and almost no flares larger than $10^{4} \mathrm{~m}^{2}$ on any two dates. The scatterplots are truncated at $10^{5} \mathrm{~m}^{2}$ to show the low end of the distribution more clearly. The rank-size plots of the flare area distributions of the full study area are shown at the bottom left of Figure 8. On all three dates, the flare population scales similarly, despite the smaller number of flares on $9 / 14$. The rank-size plots have slopes $<-1$, indicating that the distributions are dominated by large numbers of small flares. The temporal persistence map, derived from the decision tree classification (Figure 8, top), illustrates the large number of flares of varying size and persistence across these three dates.

[Figure 8 approximately here] 


\section{Discussion}

Combining horizontal drilling with hydraulic fracturing is a relatively new technological advancement in oil and gas production that has drastically increased production from tight formations (EIA 2016a). In 2015, tight oil production surpassed conventional non-tight oil in the USA, occupying the majority share of oil production, and is expected to continue doing so (EIA 2017). Production from tight formations typically involves drilling closely-spaced wells. For shale formations, production from each well is high initially, and declines steeply during its first one to two years to a low consistent level (EIA 2016b). After the initial years of production in a tight region, the number of older wells, which produce less and hence flare less, will be greater than the number of new wells added. This suggests that satellite products used to study gas flares in tight shale formations like the Bakken could benefit from Landsat's decametre resolution, to better identify individual flares that are smaller and more closely-spaced than those from conventional oil and gas production.

Decametre-resolution Landsat imagery of gas flares associated with oil production from the Bakken formation in North Dakota reveals that most of the gas flares are indeed small, densely-spaced, and persistent; moreover, these flares are not detected by either VIIRS DNB or the Nightfire product. This suggests that the majority of gas flared from this and other tight formations, where wells tend to be drilled close to each other, is not detected by hectometreresolution sensors like VIIRS. If small flares in these settings are generally persistent on time scales of months, as suggested by the bivariate distributions in Figure 8, intermittent Landsat OLI acquisitions could provide complementary estimates of small flare volumes undetected by hectometre-resolution sensors like VIIRS. Moreover, because the distance between flares (fired from different sources) is greater than $30 \mathrm{~m}$, detections within a pixel on different dates can 
reliably be treated as the same flare. This is not true with products of hectometre resolution, because new flares could be started within the same IFOV at a later date. Temporal analyses of individual flares that are closely spaced would therefore require decametre-resolution data provided by Landsat.

If nocturnal acquisitions of Landsat imagery become operational in the future, those acquisitions could be useful in identifying gas flares in densely-drilled tight formations like those in North Dakota and south Texas. Landsat could supplement studies of flares that use lowerresolution operational imagery. For higher frequency analysis, nocturnal Landsat data could also be combined with images captured by other decametre-resolution optical sensors, such as those aboard Sentinel 2 and Worldview-3 to effectively shorten revisit periods. As production of oil resources from tight formations increases worldwide (EIA 2015), and monitoring these emissions increases in importance, observations of smaller, more closely-spaced gas flares using decametre-resolution imagery may become a necessary supplement to lower-resolution sensors.

The emissions contribution of large numbers of undetected small flares could be significant, particularly if tight oil production is increased globally. Masnadi et al. (2018) find that flaring accounts for a significant, yet reducible, fraction of the carbon intensity associated with the production of fossil fuels. They report that the World Bank Global Gas Flaring Reduction (GGFR) partnership has found a nearly continuous increase in global gas flaring between 2010 and 2016. However, these estimates are based, in part, on VIIRS observations provided by NOAA, suggesting that they may be underestimates if the contribution of large numbers of small flares are not detected. If satellite monitoring of flaring is to be used operationally going forward, it will be important to account for the contribution of smaller flares not detected by hectometre-resolution sensors like VIIRS. 


\section{Acknowledgements}

R.L. thanks the School of International and Public Affairs at Columbia University for its support of her Ph.D. studies, of which this project is a part. C.S. acknowledges the support of the NASA Multi-Source Land Imaging Program (grant NNX15AT65G). 


\section{References}

Anejionu, Obinna Chukwubuikem Diony, George Alan Blackburn, and J. Duncan Whyatt. 2014. "Satellite Survey of Gas Flares Development and Application of a Landsat Based Technique in the Niger Delta". International Journal of Remote Sensing 35 (5): 19001925. doi: 10.1080/01431161.2013.879351.

Anejionu, Obinna Chukwubuikem Diony, George Alan Blackburn, and J. Duncan Whyatt. 2015. "Detecting Gas Flares and Estimating Flaring Volumes at Individual Flow Stations Using MODIS Data." Remote Sensing of the Environment 158: 81-94.

Barsi, Julia, John Barker, and John Schott. 2003. An Atmospheric Correction Parameter Calculator for a single thermal band earth-sensing instrument. In Proceedings of the 2003 IEEE International Geoscience and Remote Sensing Symposium, Toulouse, France, 2125 July 2003, 5: 3014-3016.

Casadio, Stefano, Olivier Arino, and Danilo Serpe. 2012. "Gas Flaring Monitoring from Space Using the ATSR Instrument Series." Remote Sensing of the Environment 116: 239-249. Chowdhury, Subir, Todd Shipman, Dennis Chao, Christopher D. Elvidge, Mikhail Zhizhin, and Feng-Chi Hsu. 2014. "Daytime Gas Flare Detection Using Landsat-8 Multispectral Data." In Proceedings of the IEEE International Geoscience Remote Sensing Symposium 258-261. Quebec City: IEEE. doi: 10.1109/IGARSS.2014.6946406.

Elvidge, Christopher D., Kimberly Baugh, Mikhail Zhizhin, Feng-Chi Hsu, and Tilottama Ghosh. 2017. "VIIRS Night-time Lights." International Journal of Remote Sensing 38 (21): 5860-5879.

Elvidge, Christopher D., Mikhail Zhizhin, Kimberly Baugh, Feng-Chi Hsu, and Tilottama Ghosh. 2016. "Methods for Global Survey of Natural Gas Flaring from Visible Infrared Imaging Radiometer Suite Data.” Energies 9 (1): 14. doi:10.3390/en9010014.

Elvidge, Christopher D., Mikhail Zhizhin, Kimberly Baugh, Feng-Chi Hsu, and Tilottama Ghosh. 2019. "Extending Nighttime Combustion Source Detection Limits with Short Wavelength VIIRS Data.” Remote Sensing 11 (4): 395. doi:10.3390/rs 11040395. Elvidge, Christopher D., Mikhail Zhizhin, Feng-Chi Hsu, and Kimberly Baugh. 2013. "VIIRS

Nightfire: Satellite Pyrometry at Night." Remote Sensing 5: 4423-4449. doi:10.3390/rs5094423. 
Elvidge, Christopher D., Mikhail Zhizhin, Feng-Chi Hsu, Kimberly Baugh, M Rokhis

Khomarudin, Yenni Vetrita, Parwati Sofan, Suwarsono, and Dadang Hilman. 2015.

"Long-wave Infrared Identification of Smoldering Peat Fires in Indonesia with Nighttime Landsat Data." Environmental Research Letters 10: 065002. doi:10.1088/17489326/10/6/065002.

Elvidge, Christopher D., Daniel Ziskin, Kimberly E. Baugh, Benjamin T. Tuttle, Tilottama Ghosh, Dee W. Pack, Edward H. Erwin, and Mikhail Zhizhin. 2009. “A Fifteen Year Record of Global Natural Gas Flaring Derived from Satellite Data." Energies 2 (3): 595622. doi:10.3390/en20300595.

EIA (Energy Information Administration). 2015. "Four Countries Added to Global Shale Oil and Natural Gas Resource Assessment.” Accessed 14 February 2019. https://www.eia.gov/todayinenergy/detail.php?id=24132.

EIA (Energy Information Administration). 2016a. "Wells Drilled Since Start of 2014 Provided Nearly Half of Lower 48 Oil Production in 2015.” Accessed 14 February 2019. https://www.eia.gov/todayinenergy/detail.php?id=25472.

EIA (Energy Information Administration). 2016b. "Initial Production Rates in Tight Oil Formations Continue to Rise.” Accessed 14 February 2019. https://www.eia.gov/todayinenergy/detail.php?id=24932.

EIA (Energy Information Administration). 2017. "Tight Oil Expected to Make Up Most of U.S. Oil Production Increase Through 2040.” Accessed 14 February 2019. https://www.eia.gov/todayinenergy/detail.php?id=29932.

Fisher, Daniel, and Martin J. Wooster. 2018. "Shortwave IR Adaption of the Mid-Infrared Radiance Method of Fire Radiative Power (FRP) Retrieval for Assessing Industrial Gas Flaring Output." Remote Sensing 10 (2): 305. doi:10.3390/rs10020305.

Masnadi, Mohammad S., Hassan M. El-Houjeiri, Dominik Schunack, Yunpo Li, Jacob G. Englander, Alhassan Badahdah, Jean-Christophe Monfort, et al. 2018. "Global Carbon Intensity of Crude Oil Production.” Science 361 (6405): 851-853. doi:10.1126/science.aar6859.

NDIC (North Dakota Industrial Commission). 2018. "ND Monthly Oil Production Statistics." 
Accessed 16 July 2018. https://www.dmr.nd.gov/oilgas/stats/historicaloilprodstats.pdf. NDIC (North Dakota Industrial Commission). 2019. "Historical Monthly Gas Production and Sales Statistics.” Accessed 15 February 2019. https://www.dmr.nd.gov/oilgas/stats/Gas1990ToPresent.xls.

Schroeder, Wilfrid, Patricia Oliva, Louis Giglio, Brad Quayle, Eckehard Lorenz, and Fabiano Morelli. 2016. “Active Fire Detection Using Landsat-8/OLI Data.” Remote Sensing of the Environment 185: 210-220. doi:10.1016/j.rse.2015.08.032. 